\title{
ATENÇÃO ONLINE DE ARTIGOS DO PORTAL PERIÓDICOS UFMG: ANÁLISE DOS DADOS DO FACEBOOK
}

Ronaldo Ferreira Araujo'; Tiago Rodrigo Marçal Murakami²; Sergio Dias Cirino³; Carla Cristina Vieira de Oliveira ${ }^{4}$

\section{Como citar:}

ARAUJO, R. F.; MURAKAMI, T. R. M.; CIRINO, S. D.; OLIVEIRA, C. C. V de. Atenção online de artigos do portal periódicos ufmg: análise dos dados do facebook. In: ABEC MEETING, 1, 2017, Curitiba. Anais...

Curitiba: Associação Brasileira de Editores Científicos, 2017. p. 66-72.

http://dx.doi.org/10.21452/abecmeeting.2017.012

Resumo: Discorre sobre dados de interação do Facebook como indicativo de atenção online e métricas alternativas da produção científica. Analisa por meio de uma pesquisa exploratória o impacto social de artigos de revistas científicas do Portal Periódicos UFMG segundo seus dados de curtidas, compartilhamentos e comentários. Foram analisados 46 periódicos do portal com publicação no período de 2010 a 2017, os quais forneceram 6.437 artigos, sendo que apenas 543 deles possuíam dados de interação. A atenção online é caracterizada segundo sua distribuição por faixa de interação, área de conhecimento, período e analisada pelo desempenho de revistas e artigos.

Palavras-chave: Altmetria. Atenção online. Facebook. Portal Periódicos UFMG.

\begin{abstract}
Discusses interaction data from Facebook as indicative of online attention and altmetrics of scientific production. It analyzes based on an exploratory study the social impact of articles of the UFMG scientific journals gateway according to its data of tastings, shares and comments. We analyzed 46 journals with publication in the period from 2010 to 2017, which provided 6,437 articles, and only 543 of them had interaction data. Online attention is characterized according to its distribution by range of interaction, area of knowledge, period and analyzed by the performance of journals and articles.
\end{abstract}

Keywords: Altmetria. Online attention. Facebook. UFMG scientific journals gateway.

\section{INTRODUÇÃO}

A avaliação do impacto social por meio de métricas alternativas de publicações de resutados parciais ou finais de pesquisa tem sido cada vez mais buscada pela comunidade interessada em complementar os estudos tradicionais de avaliações de desempenho com base em indicadores quantitativos específicos (ex. produtividade científica, número de citações e fatores de impacto) que trazem consigo inúmeras críticas, sobretudo devido a sua negligência dos aspectos qualitativos e subjetivos do desempenho científico.

No contexto de uma ciência aberta que se utiliza dos recursos digitais para potencialização das atividades de pesquisa a fim de que estas ocorram de forma mais democrática e participativa, a altmetria (altmetrics) se apresenta como um campo promissor para se pensar essa complementariedade e pode contribuir no levantamento de indicadores que auxiliam na compreensão do interesse público pela ciência ao atentar sobre a circulação da informação científica na web social e perceber as métricas das interações que ela gera.

Devido ao grande número de mídias sociais e plataformas como Facebook, Twitter, YouTube, Wikipédia e outros que passam a ser consideradas como fonte de dados 
altmétricos, e tendo em vista que possuem suas particularidades, tem sido cada vez mas necessário estudos que compreendem o que cada uma delas representa para a comunicação e divulgação científica.

Diante disso o presente estudo tem por objetivo refletir sobre uma dessas mídias, o Facebook, compreender as interações deste dispositivo informacional, a saber, as curtidas, compartilhamentos e comentários, enquanto elementos de atenção online e fonte de dados altmétricos, e dialogar tais reflexões com uma análise empírica que considerou revistas científicas do Portal Periódicos UFMG.

As interações ou ações de curtir, compartilhar e comentar no Facebook são vistas como apropriações simbólicas e podem indicar intenções distintas. Cientistas e pesquisadores quando "curtem" algo dão visibilidade e audiência a conteúdos relevantes, o que pode ser considerado um indicador valioso do impacto de trabalhos acadêmicos, uma ação positiva de gostar de um conteúdo e consequentemente transmiti-lo para sua rede (RINGELHAN; WOLLERSHEIM; WELPE, 2015) o que pode ser estendido às ações de compartilhar e de comentar.

O compartilhamento no Facebook pode ser considerado um bom filtro de informação funcionando como ações de recomendações e o comentário expressa um indicativo de receptividade podendo indicar avaliações e debates em torno de artigos recém-publicados. Assim, "artigos bastante compartilhados logo após sua publicação são aqueles de grande apelo para uma área" (IAMARINO, 2013) e os mais "comentados" enfatizam que o apelo tem mobilizado a discussão (BORNMANN, 2014).

\section{MATERIAL E MÉTODO}

Pesquisa exploratória tendo como universo empírico artigos de revistas científicas presentes no Portal Periódicos UFMG. O portal foi criado em 2015 e atualmente conta com 63 revistas, das quais 57,6\% não possui um identificador permanente, o Digital Object Indentifier - DOI. Para contornar a ausência desse padrão comum de identificação exigido pelos serviços atuais de aferição de métricas alternativas, a coleta de dados altmétricos do Facebook seguiu as orientações metodológicas de Araújo, Murakami e Andrade (2016) na utilização de uma solução via Application Program Interface (API) que considera apenas os periódicos suportados pelo protocolo OAI-PHM utilizado no SEER/OJS, o que correspondeu a 52 revistas, das quais seis foram desconsideradas por apresentarem erro na versão do SEER/OJS e impedirem a coleta dos metadados da produção. 
Sendo assim foram coletados e analisados na última quinzena de outubro de 2017 os artigos de 46 revistas do portal tendo como recorte temporal os anos de 2010 a 2017, uma vez que a literatura expressa que a altmetria é adequada para aferição de impacto de publicações recentes. As consultas foram parametrizadas pelas URLs principal e secundária de todos os artigos das 46 revistas, com sua representação quantitativa em termos de "curtidas", "compartilhamentos" e "comentários". Os dados são apresentados segundo o volume quantitativo por área de conhecimento, por revistas para em seguida discorrer sobre os artigos com maior atenção online.

\section{DISCUSSÃO DOS RESULTADOS}

No período analisado (2010 a 2017) os 46 periódicos do portal somavam 6.437 artigos, dos quais apenas $543(8,43 \%)$ obtiveram ao menos uma interação no Facebook. Os 543 artigos forneceram para análise um total de 11.265 dados de atenção dando uma média de 20,74\% de interação por artigo. A Tabela 1 apresenta a distribuição dos artigos por faixa de interação e a Tabela 2 por período de interação (dois anos).

Tabela 1. Artigos por faixa de interação

\begin{tabular}{|l|r|r|}
\hline Faixa de interação & Artigos & (\%) \\
\hline Mais de 100 & 24 & 4,42 \\
\hline De 50 a 99 & 38 & 7,00 \\
\hline De 10 a 49 & 156 & 28,73 \\
\hline Menos de 10 & 325 & 59,85 \\
\hline Total & $\mathbf{5 4 3}$ & $\mathbf{1 0 0}$ \\
\hline \multicolumn{2}{|c|}{ Fonte: dados da pesquisa (2017). } \\
\hline
\end{tabular}

Tabela 2. Artigos por período de interação

\begin{tabular}{|l|r|r|}
\hline Anos & Artigos & $\mathbf{( \% )}$ \\
\hline $2010-2011$ & 47 & 8,66 \\
\hline $2012-2013$ & 141 & 25,97 \\
\hline $2014-2015$ & 148 & 27,26 \\
\hline $2016-2017$ & 207 & 38,12 \\
\hline Total & $\mathbf{5 4 3}$ & $\mathbf{1 0 0}$ \\
\hline \multicolumn{2}{|c|}{ Fonte: dados da pesquisa (2017). } \\
\hline
\end{tabular}

É possível perceber que a maioria dos artigos obteve menos de 10 interações $(59,8 \%)$ e que só um número reduzido alcançou mais de 100 interações (4,4\%). No que se refere a essa distribuição pelos anos analisados verificamos um movimento crescente que confere a premissa de que artigos recém-publicados costumam atingir uma maior pontuação altmétrica.

A distribuição da atenção online obtida no Facebook por área de conhecimento indica a Ciências Sociais Aplicadas com 49,9\% do total, seguido da área de Linguística, Letras e Artes com 29,4\%, das Ciências da Saúde com 7,03\% e das Ciências Humanas e Exatas e da Terra, com 6,85\% e 6,42\%, respectivamente. A área Interdisciplinar e as Ciências Agrárias tiveram o menor desempenho com 0,31 \% e 0,04\% e as Engenharias não apresentou nenhuma incidência.

Os 11.265 dados altmétricos refletem uma atenção online distribuída em 8.233 curtidas (C1), 1.493 compartilhamentos (C2) e 1.539 comentários (C3) obtidos pelos 
ARAÚJO, R. F.; MURAKAMI, T. R. M.; CIRINO, S. D.; OLIVEIRA, C. C. V.

"Atenção online de artigos do portal periódicos ufmg: análise dos dados do facebook"

periódicos, cuja a distribuição consta representada na Tabela 3, ordenados pela posição (\#) do periódico segundo seu desempenho na soma total das interações (Para facilitar a visualização as revistas com até 99 interações foram contabilizadas, mas não constam nominadas na tabela).

Tabela 3 - Distribuição dos periódicos por interação no Facebook

\begin{tabular}{lrrrrr}
\hline Nome do Periódico & C1 & C2 & C3 & Total & $\mathbf{( \% )}$ \\
\hline Perspectivas em Ciência da Informação & 1071 & 323 & 252 & 1646 & 14,61 \\
Revista da Faculdade de Direito da UFMG & 1082 & 135 & 102 & 1319 & 11,71 \\
Revista Brasileira de Estudos Políticos & 950 & 147 & 97 & 1194 & 10,60 \\
PÓS: Rev. Prog. Pós-graduação Artes da EBA/UFMG & 522 & 79 & 68 & 669 & 5,94 \\
Revista Geonomos & 453 & 38 & 80 & 571 & 5,07 \\
Texto Livre: Linguagem e Tecnologia & 353 & 36 & 173 & 562 & 4,99 \\
LICERE - Rev. Prog. Pós-graduação Interdisc. Est. Lazer & 391 & 50 & 50 & 491 & 4,36 \\
Em Tese & 335 & 74 & 80 & 489 & 4,34 \\
Aletria: Revista de Estudos de Literatura & 311 & 68 & 66 & 445 & 3,95 \\
Revista Docência do Ensino Superior & 290 & 37 & 41 & 368 & 3,27 \\
Arquivo Maaravi: Rev. Dig. Est. Judaicos da UFMG & 241 & 64 & 53 & 358 & 3,18 \\
Revista Brasileira de Estudos do Lazer & 224 & 21 & 59 & 304 & 2,70 \\
Cadernos Benjaminianos & 199 & 38 & 51 & 288 & 2,56 \\
Revista do Centro Acadêmico Afonso Pena & 197 & 68 & 23 & 288 & 2,56 \\
O Eixo e a Roda: Revista de Literatura Brasileira & 223 & 22 & 25 & 270 & 2,40 \\
Gestão e Sociedade & 145 & 28 & 32 & 205 & 1,82 \\
Múltiplos Olhares em Ciência da Informação & 134 & 29 & 37 & 200 & 1,78 \\
Revista Geografias & 104 & 33 & 25 & 162 & 1,44 \\
Temporalidades & 122 & 11 & 29 & 162 & 1,44 \\
Lamparina - Revista de Ensino de Artes Cênicas & 77 & 41 & 27 & 145 & 1,29 \\
Nova Economia & 106 & 12 & 9 & 127 & 1,13 \\
Revista Teoria \& Sociedade & 83 & 20 & 10 & 113 & 1,00 \\
Revista Ágora: pol. públicas, comunic. e gov. inf. & 59 & 7 & 45 & 111 & 0,99 \\
Revista de Ciências do Estado & 85 & 9 & 12 & 106 & 0,94 \\
Revista Multiface Online & 81 & 8 & 13 & 102 & 0,91 \\
\hline De 50 a 99 interações (5 Revistas) & 285 & 61 & 51 & 397 & 3,52 \\
\hline Até 50 interações (10 revistas) & 110 & 34 & 29 & 173 & 1,54 \\
\hline Nenhuma interação (4 revistas) & 0 & 0 & 0 & 0 & 0,00 \\
\hline Total & $\mathbf{8 2 3 3}$ & $\mathbf{1 4 9 3}$ & $\mathbf{1 5 3 9}$ & $\mathbf{1 1 2 6 5}$ & $\mathbf{1 0 0}$ \\
\hline & & & & &
\end{tabular}

A revista "Perspectivas em Ciência da Inf." alcançou maior audiência na atenção online com 1.646 dados de interação (14,61\%), seguida da "Revista da Faculdade de Direito da UFMG" com 1.319 (11,71\%) e da "Revista Brasileira de Estudos Políticos" com 1.194 $(10,60 \%)$. As ações de "curtir" representam o tipo de interação mais evidente do universo analisado, corresponde a 73,08\% das interações. Os compartilhamento e comentários tiverem desempenho aproximado e representam 13,66\% e 13,25\%, respectivamente. A ação de curtir e o compartilhar é justamente aquela ligação ao movimento de legitimar o que está sendo dito, 
ARAÚJO, R. F.; MURAKAMI, T. R. M.; CIRINO, S. D.; OLIVEIRA, C. C. V.

"Atenção online de artigos do portal periódicos ufmg: análise dos dados do facebook"

sinalizando concordância e costumam ser associados a uma ação positiva em relação ao conteúdo e o comentário equivale dizer que há uma possível a discussão em torno dos artigos (ARAÚJO; MURAKAMI, 2016).

Para uma melhor aproximação do universo analisado, tendo em vista o volume de artigos, foi realizado um recorte com os dez mais expressivos, ou seja, um ranking com os artigos que obtiveram os maiores índices altmétricos. Tais artigos podem ser visualizados na Tabela 4 com descrição do título, seus autores, título do periódico, ano de publicação e o número de curtidas $(\mathrm{C} 1)$, compartilhamentos $(\mathrm{C} 2)$ e comentários $(\mathrm{C} 3)$.

Tabela 4 - Dez artigos que obtiveram mais atenção online.

\begin{tabular}{|c|c|c|c|c|c|c|c|}
\hline Título & Autores & Periódico & Ano & C1 & $\mathrm{C} 2$ & C3 & Total \\
\hline Direito, figura do ódio & $\begin{array}{l}\text { José Rodrigo } \\
\text { Rodriguez }\end{array}$ & $\begin{array}{l}\text { Revista } \\
\text { Brasileira de } \\
\text { Estudos } \\
\text { Políticos }\end{array}$ & 2013 & 255 & 59 & 34 & 348 \\
\hline $\begin{array}{l}\text { Aspectos macroscópicos dos } \\
\text { esteatitos encontrados no } \\
\text { Santuário Bom Jesus do } \\
\text { Matosinhos, Congonhas/MG }\end{array}$ & $\begin{array}{l}\text { Mônica Pessoa Neves; } \\
\text { Antônio Gilberto } \\
\text { Costa; Úrsula de } \\
\text { Azevedo Ruchkys }\end{array}$ & $\begin{array}{l}\text { Revista } \\
\text { Geonomos }\end{array}$ & 2016 & 263 & 1 & 54 & 318 \\
\hline $\begin{array}{l}\text { Autobiografia na cena } \\
\text { contemporânea: tensionamentos } \\
\text { entre o real e o ficcional }\end{array}$ & $\begin{array}{l}\text { Gabriela Lirio Gurgel } \\
\text { Monteiro }\end{array}$ & $\begin{array}{l}\text { PÓS: Rev. } \\
\text { Prog. Pós- } \\
\text { graduação } \\
\text { Artes da } \\
\text { EBA/UFMG. }\end{array}$ & 2016 & 280 & 11 & 10 & 301 \\
\hline $\begin{array}{l}\text { Musil e Benjamin: a ética do } \\
\text { homem sem qualidades e o } \\
\text { empobrecimento da experiência }\end{array}$ & $\begin{array}{l}\text { Juliano Garcia } \\
\text { Pessanha; Luciana } \\
\text { Araújo Marques }\end{array}$ & $\begin{array}{l}\text { Cadernos } \\
\text { Benjaminianos }\end{array}$ & 2016 & 158 & 9 & 21 & 188 \\
\hline $\begin{array}{l}\text { A dramaturgia de Lourdes } \\
\text { Ramalho como expressão da } \\
\text { modernidade teatral brasileira }\end{array}$ & $\begin{array}{l}\text { Diogenes Andre Vieira } \\
\text { Maciel }\end{array}$ & $\begin{array}{l}\text { O Eixo e a } \\
\text { Roda: Revista } \\
\text { de Literatura } \\
\text { Brasileira }\end{array}$ & 2017 & 154 & 11 & 17 & 182 \\
\hline $\begin{array}{l}\text { Para que serve a dupla avaliação } \\
\text { cega por pares? Poder estatal e } \\
\text { autorregulação na avaliação dos } \\
\text { Programas de Pós-Graduação }\end{array}$ & $\begin{array}{l}\text { José Rodrigo } \\
\text { Rodriguez }\end{array}$ & $\begin{array}{l}\text { Revista } \\
\text { Brasileira de } \\
\text { Estudos } \\
\text { Políticos }\end{array}$ & 2013 & 153 & 21 & 0 & 174 \\
\hline $\begin{array}{l}\text { Sistema eleitoral, corrupção e } \\
\text { reforma política }\end{array}$ & $\begin{array}{l}\text { Bruno Pinheiro } \\
\text { Wanderley Reis }\end{array}$ & $\begin{array}{l}\text { Revista do } \\
\text { Centro } \\
\text { Acadêmico } \\
\text { Afonso Pena }\end{array}$ & 2014 & 114 & 29 & 17 & 160 \\
\hline $\begin{array}{l}\text { Os significados da prática e da } \\
\text { assistência do futebol amador para } \\
\text { frequentadores de um clube da } \\
\text { cidade de Piracicaba/SP }\end{array}$ & $\begin{array}{l}\text { Milena Avelaneda } \\
\text { Origuela; Cinthia } \\
\text { Lopes da Silva }\end{array}$ & $\begin{array}{l}\text { Revista } \\
\text { Brasileira de } \\
\text { Estudos do } \\
\text { Lazer }\end{array}$ & 2017 & 114 & 3 & 35 & 152 \\
\hline $\begin{array}{l}\text { Caracterização da Prática } \\
\text { Esportiva/Recreativa do Skate em } \\
\text { São Paulo, Paraná e Rio Grande do } \\
\text { Sul }\end{array}$ & $\begin{array}{l}\text { Luana Mari Noda; } \\
\text { Giuliano Gomes de } \\
\text { Assis Pimentel }\end{array}$ & $\begin{array}{l}\text { LICERE - } \\
\text { Rev. Prog. } \\
\text { Pós-graduação } \\
\text { Interdisc. Est. } \\
\text { Lazer }\end{array}$ & 2015 & 120 & 20 & 4 & 144 \\
\hline $\begin{array}{l}\text { Friedrich Engels e o duplo aspecto } \\
\text { da igualdade }\end{array}$ & Vitor Bartoletti Sartori & $\begin{array}{l}\text { Revista da } \\
\text { Faculdade de } \\
\text { Direito da } \\
\text { UFMG }\end{array}$ & 2016 & 130 & 4 & 4 & 138 \\
\hline
\end{tabular}

Fonte: dados da pesquisa (2017). 
Conforme esperado de indicadores altmétricos, artigos mais recentes tendem a obter maior atenção online, e consequentemente uma pontuação elevada (ARAUJO; MURAKAMI, 2016). Os artigos apresentados na tabela foram publicados nos anos de 2013 (2), 2014 (1), 2015 (1), 2016 (4) e 2017 (2). Artigos dos anos de 2010 a 2012 tiveram baixa ou nenhuma expressão nas primeiras 100 posições. Mais uma vez os dados de "curtidas" superam consideravelmente os de "compartilhamento" e "comentários".

\section{CONSIDERAÇÕES FINAIS}

Serviços e soluções disponíveis no mercado para aferição de métricas alternativas para produtos da ciência (livros, artigos, revistas, repositórios institucionais, etc) consideram várias fontes de dados de plataformas, mídias sociais e gerenciadores de referências; exigem padrões para seu monitoramento, e; requerem um investimento e custo relativamente alto que nem sempre as revistas dispõem de recursos para aplicar.

No primeiro aspecto é necessário melhor compreensão de cada fonte e as implicações de seu uso para a comunicação científica, no segundo a preocupação é quanto a infraestrutura de informação de pesquisa e seus aspectos técnicos e normativos, ficando no terceiro, reflexões quanto ao fomento e sustentabilidade financeira de revistas.

No estudo apresentado consideramos, mesmo que de forma preliminar, que atendemos aos três aspectos com a solução utilizada na pesquisa. Primeiro por nos dedicarmos á compreensão e análise de uma fonte, no caso o Facebook. No segundo caso contorna-se a ausência de identificadores como o DOI com consultas por URL via Facebook API que não demanda custo, embora exige um pouco de conhecimento técnico para as parametrizações. O total de quatro revistas não apresentou dados altmétricos quando consultadas pelas URLs dos seus artigos, o que precisa ser melhor investigado.

Consideramos que as interações com conteúdo de artigos científicos no Facebook como como ricas fontes de dados altmétricos, as quais podem agregar informações de impacto social das publicações das revistas do Portal Periódicos UFMG fornecendo uma visão adicional sobre o desempenho científico dos artigos quando curtidos, compartilhados ou comentados.

\section{REFERÊNCIAS}

ARAUJO, R. F.; MURAKAMI, T. R. M. ; ANDRADE, R. L. V. . Repertório da produção periódica brasileira de Ciência da Informação: indícios embrionários. Pesquisa Brasileira em Ciência da Informação e Biblioteconomia, v. 11, p. 44-53, 2016. 
ARAUJO, R. F.; MURAKAMI, T. R. M. . Atenção online de artigos de ciência da informação: análise a partir de dados altmétricos do Facebook. In: Encontro Brasileiro de Bibliometria e Cientometria, v.5, 2016, São Paulo. Anais... São Paulo: ECA/USP, 2016. https://doi.org/10.6084/m9.figshare.3479249.v1

BORNMANN, L. Do altmetrics point to the broader impact of research? An overview of benefits and disadvantages of altmetrics. Journal of Informetrics, v.8, n.4, p.895-903, out., 2014. https://doi.org/10.1016/j.joi.2014.09.005

IAMARINO, A. Você compartilha, eu curto e nós geramos métricas. SciELO em Perspectiva. Ago., 2013.

RINGELHAN, S.; WOLLERSHEIM, J.; WELPE, I. M. I Like, I Cite? Do Facebook Likes Predict the Impact of Scientific Work? PLoS ONE, v.10, n.8, ago., 2015. https://doi:10.1371/journal.pone.0134389 